\title{
Magnetic turbulence amplification through nonresonant Bell's instability in shock precursors of young supernova remnants
}

\author{
Oleh Kobzar* \\ Instytut Fizyki Jqadrowej PAN, \\ ul. Radzikowskiego 152, 31-342 Kraków, Poland \\ E-mail: oleh.kobzar@ifj.edu.pl \\ Jacek Niemiec \\ Instytut Fizyki Jąrowej PAN, \\ ul. Radzikowskiego 152, 31-342 Kraków, Poland
}

Martin Pohl

Institute of Physics and Astronomy, University of Potsdam,

Karl-Liebknecht-Strasse 24/25, 14476 Potsdam, Germany

DESY, Platanenallee 6, 15738 Zeuthen, Germany

Artem Bohdan

Instytut Fizyki Jadrowej PAN,

ul. Radzikowskiego 152, 31-342 Kraków, Poland

\begin{abstract}
In the present work we study the cosmic-ray-current-driven nonresonant Bell's instability operating upstream of a young supernova remnant shocks with use of a very-large scale 2D fully kinetic Particle-In-Cell simulations. In contrast to earlier simulation works that used periodic simulation boxes here for the first time we apply a new realistic setup with open boundaries in the CR drift direction, which accounts for mass conservation in decelerating flows. This setup allows us to investigate both the temporal and the spatial development of the instability. The results demonstrate magnetic-field amplification to nonlinear amplitudes, as expected on the basis of earlier studies with periodic simulation boxes. The effects of backreaction on CRs that slow down the initial ambient plasma-to-CR relative drift velocity, limit further growth of the turbulence and lead to its saturation are also re-confirmed. We discuss new features observed in the flow that provide additional field amplification through plasma compression and filamentation. We show that electromagnetic turbulence inelastically scatters CRs and strongly modifies their distribution. Spatial CR scattering in the precursor is compatible with Bohm diffusion. The turbulence also leads to the strong nonadiabatic heating of the ambient plasma. Ion distributions show supra-thermal tails resulting from stochastic scattering in the turbulent electric fields.
\end{abstract}

35th International Cosmic Ray Conference - ICRC2017

10-20 July, 2017

Bexco, Busan, Korea

* Speaker. 


\section{Introduction}

Shock waves of young shell-type supernova remnants are widely believed to be acceleration sites of Galactic cosmic rays (CRs) with energies up to a few peta-electronvolts. The diffusive shock acceleration process requires turbulent magnetic field in the shock precursor, amplified to a level much higher than typical for the interstellar medium. Upstream magnetic field can be amplified by the CRs themselves. It was shown by Bell [1], that nonresonant CR current-driven instability provides the most rapid turbulent field growth on scales much smaller than the CR gyroradii. The fastest growth occurs for wavevector aligned with the mean homogeneous magnetic field $\mathbf{B}_{\mathbf{0}}$ at the rate $\gamma_{\max }=v_{\mathrm{A}} k_{\| \max }$, where $v_{\mathrm{A}}$ is the Alfvén velocity and the suscript "max" stands for the most unstable mode. Corresponding wavelength is then $\lambda_{\max }=4 \pi B_{\| 0} / \mu_{0} j_{\| \mathrm{CR}}$, where $B_{\| 0}$ is the magnetic field strength, $\mu_{0}$ is the magnetic permeability of space and $j_{\| \mathrm{CR}}$ is the CR current density, here is assumed to be parallel to $\mathbf{B}_{\mathbf{0}}$.

The the nonresonant Bell's instability has been widely studied with different numerical methods: MHD [1 - 4], hybrid kinetic [5, 6] and fully kinetic particle-in-cell (PIC) [7 - 9]. All these simulations used periodic boundary conditions, which allow only the temporal evolution to be studied. Application of a new realistic setup with open boundaries in our recent PIC experiment [10] has for the first time made possible to investigate the spatio-temporal development of the instability.

\section{Numerical model and simulation setup}

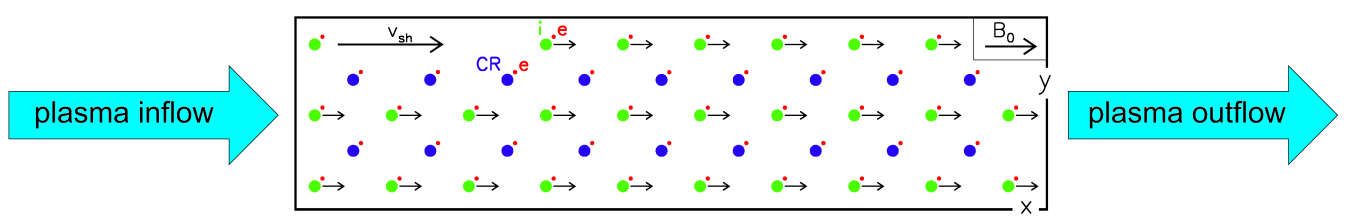

Figure 1: Sketch of the simulation setup with open boundaries in the plasma flow direction.

The numerical experiment is performed in the CR rest frame to maintain stability of the system with open boundaries (Fig. 1). In this frame, the electron-ion plasma forms a beam moving with velocity $v_{\mathrm{sh}}$ in the $x$-direction and along the mean magnetic field $B_{0}$. Charge and current balance is ensured with excess electrons initiated at rest with CRs. The simulation box size is $L_{x} \times L_{y}=$ $130,000 \times 12,000 \Delta^{2}$, where $\Delta$ is the grid cell size. In physical units $L_{x} \times L_{y} \simeq(293 \times 27) \lambda_{\max }^{2}$. All 3 components of particle velocities and electromagnetic fields are followed. The beam and CR particles initially fill up the whole computational box. The left and right boundaries are open for the ambient beam plasma and fields, but reflect CRs. Periodic boundary conditions are applied in the transverse $y$-direction.

We use 9 particle per cell per species. The electron skin depth $\lambda_{\mathrm{se}}=c / \omega_{\mathrm{pe}}=4 \Delta$, where $c$ is the speed of light and $\omega_{\mathrm{pe}}$ is the electron plasma frequency. CR ions are initially isotropic and monoenergetic with Lorentz factor $\Gamma_{\mathrm{CR}}=50$. The density ratio between plasma ions and CRs is $N_{\mathrm{i}} / N_{\mathrm{CR}}=50$. The homogeneous magnetic field, $B_{\| 0}$, is chosen for a CR gyroradius $r_{\mathrm{CRg}} \simeq$ $140,000 \Delta$ and an Alfvén velocity $v_{\mathrm{A}}=0.01 c$. The ambient plasma velocity $v_{\mathrm{sh}}=0.4 c$. The ions are in thermal equilibrium with the electrons whose initial thermal velocity is $v_{\mathrm{e}, \mathrm{th}}=0.01 \mathrm{c}$. The 


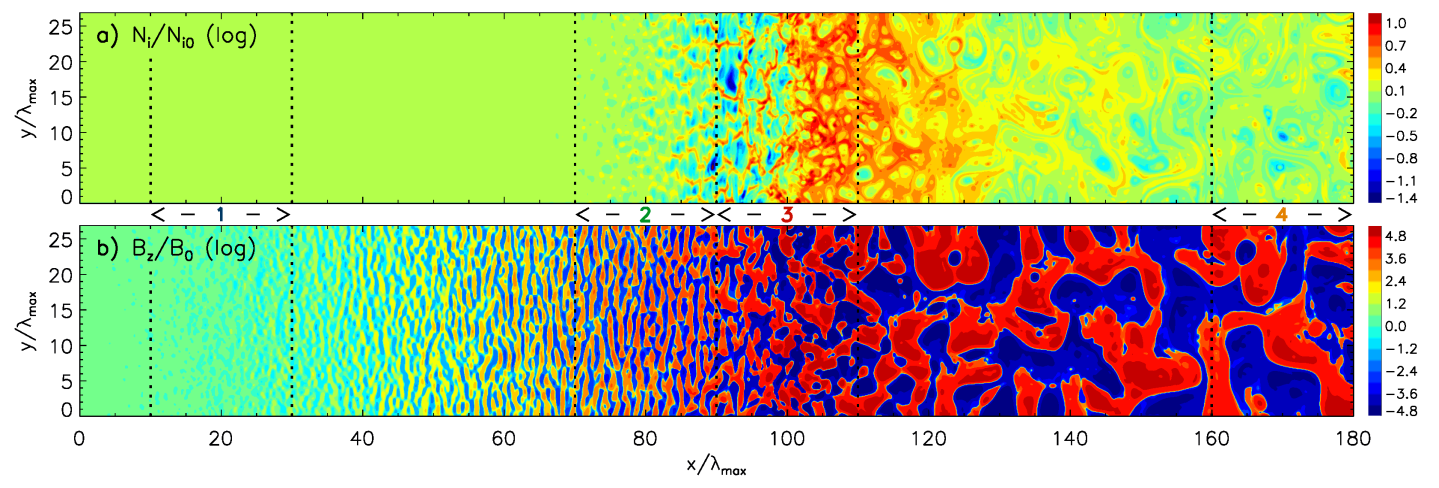

Figure 2: Distribution of the normalized ion number density $N_{\mathrm{i}} / N_{\mathrm{i} 0}$ (a) and the normalized magnetic-field component $B_{z} / B_{\| 0}$ (b) at time $t \gamma_{\max }=26.9$. Logarithmic scaling is applied for density. The scaling for $B_{z}$ is sign-preserving logarithmic, $\left(\operatorname{sgn}\left(B_{z}\right)\left(3+\log \left[\max \left(10^{-3},\left|B_{z}\right| / B_{\| 0}\right)\right]\right)\right)$. Note four color-coded regions from which particle distributions are extracted for our analysis (see Fig. 4).

ion-to-electron mass ratio is reduced to $m_{\mathrm{i}}=50 \mathrm{~m}_{\mathrm{e}}$. The wavelength of the most unstable mode is $\lambda_{\max } \simeq 444.3 \Delta$ and the inverse growth rate is $\gamma_{\max }^{-1} \simeq 14,142 \delta t$, where timestep $\delta t=0.125 / \omega_{\mathrm{pe}}$.

\section{Results}

\subsection{Global evolution of the nonresonant instability}

Figure 2 shows the normalized distribution of the ambient ion number density and transverse magnetic-field component, $B_{z}$, at the end of the simulation at $t \gamma_{\max }=26.9$. The spatial distribution from left to right, along the plasma beam flow direction reflects the temporal development of the instability that has been observed in earlier studies using periodic boxes. Magnetic turbulence appears at $x / \lambda_{\max } \approx 22$ and then grows to nonlinear wave amplitudes, reaching its saturation at $x / \lambda_{\max } \approx 90-100$. Beyond this point, for $x / \lambda_{\max } \gtrsim 100$, the waves quickly cascade towards longer wavelengths. Note, that in this turbulent zone the instability development is mainly temporal because the beam particles in this region have traveled the same distance on the CR background from the time of their initial injection.

Physical characteristics of the instability are further illustrated in Figure 3, whose left panel shows the spatial profiles of ion number density, magnetic and electric field amplitudes and particle velocities, and right panel presents the time evolution of the spatially averaged magnetic field strength in the turbulent zone. The level of magnetic field amplification in the deep turbulence zone is $\left\langle\delta B / B_{\| 0}\right\rangle \approx 15$, i.e. the same as observed in our earlier simulations with periodic boxes [9]. Amplified magnetic field strongly back-reacts on the system, modifying the bulk motion of CRs and plasma, whose relative drifts effectively converge, leading to the saturation of the instability (Fig. 3d). As a consequence of this nonlinear back-reaction, a strong density enhancement appears at $x / \lambda_{\max } \approx 100-130$ (Figs. 2 and $3 \mathrm{a}$ ). This plasma compression is revealed for the first time and results from the collision between the incoming particle beam and decelerated plasma in the turbulent zone. The compression increases by a factor of 4 , as in a strong nonrelativistic shock. An additional field amplification up to $\sim 30 \%$ is observed in this region (Fig. 3b). 

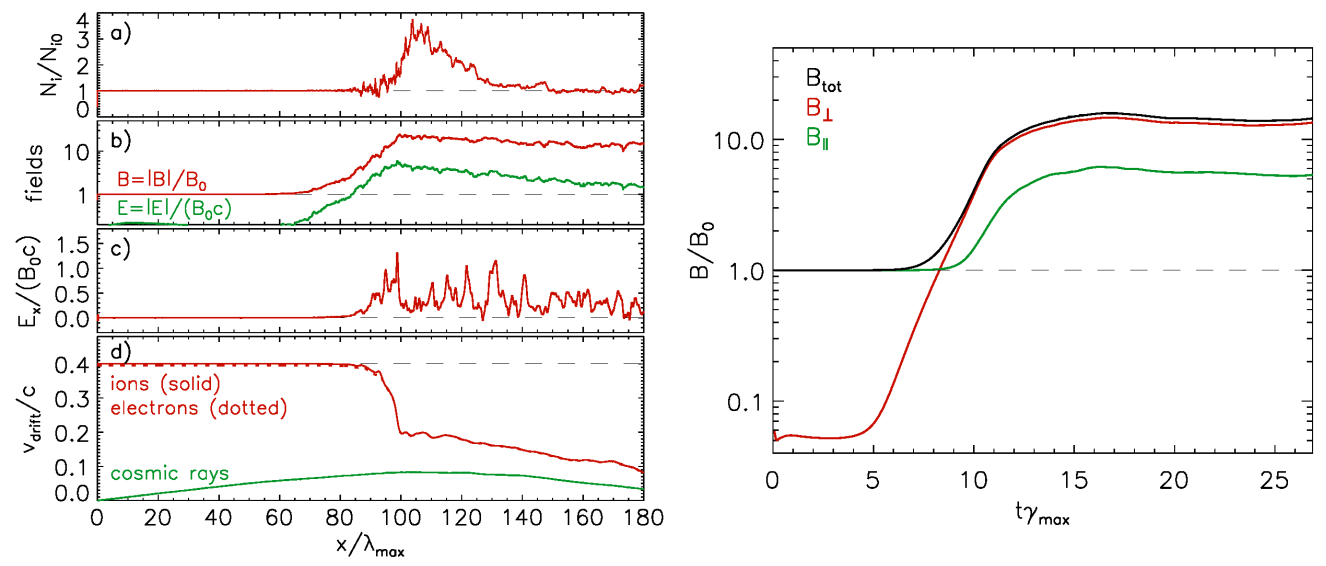

Figure 3: Left panel: spatial profiles of the ion number density (a), magnetic- and electric-field amplitudes (b), $E_{x}$ component of the electric field (c) and particle velocities (d) at time $t \gamma_{\max }=26.9$. Right panel: temporal evolution of the spatially averaged magnetic-field strength in the region $135 \leq x / \lambda_{\max } \leq 180$.

In the non-linear phase, the magnetic turbulence is accompanied by strong density and velocity fluctuations. Turbulent electric field also appears at amplitude an order of magnitude smaller than that of the magnetic field (Fig. 3b). The main part of the turbulent electric field arises from magnetic-field transport, but an electrostatic field component resulting from charge separation is also present. Because of significant transverse plasma motions, an electric-field component in plasma drift direction with non-zero mean amplitude is present in the turbulence region (Fig. 3c). Bulk energy is transferred between CRs, the ambient plasma and turbulence through work in this field. Electromagnetic fields scatter the particles and significantly modify their distributions.

The spatial structure of the plasma velocity profile can be also traced in Figure 4 that shows longitudinal phase-space distributions for electrons and ions at $t \gamma_{\max }=26.9$. Significant plasma heating is visible in the region of strong turbulence. The ambient plasma velocity slows down from $0.4 c$ to $0.15 c$ at the transition into the turbulent zone (Fig. $4 c$ ). Stronger heating is observed in the vicinity of the compression region. This structure also exhibits a distinctive feature of a supercritical shock - part of the ambient ions is reflected there (Fig. 4b-c) as a diffuse particle

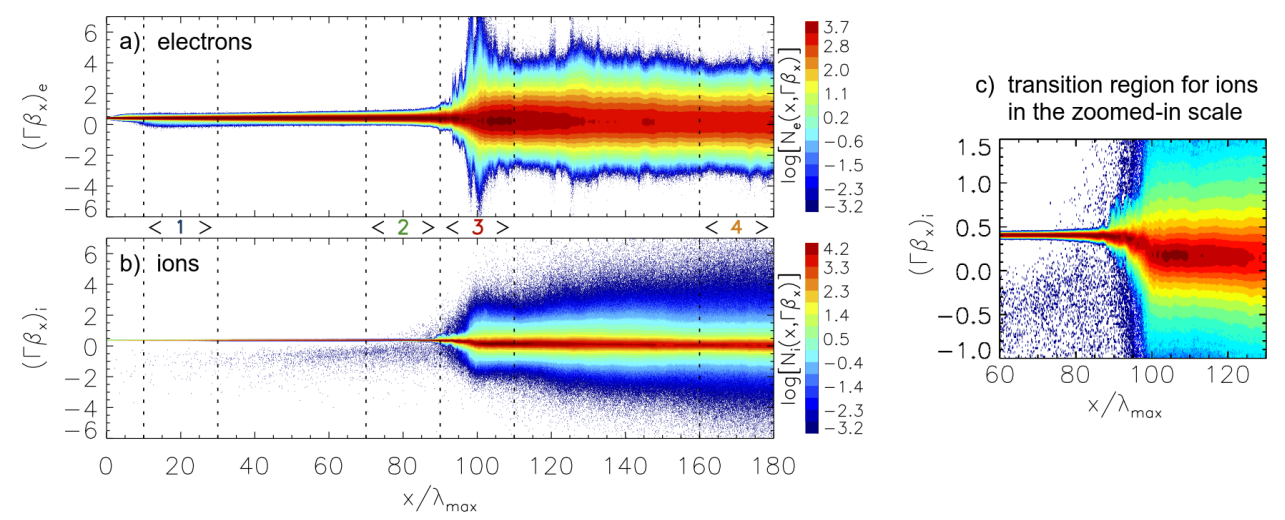

Figure 4: Phase-space distribution of the $p_{x}$-component for (a) electrons and (b, c) ions at $t \gamma_{\max }=26.9$. 

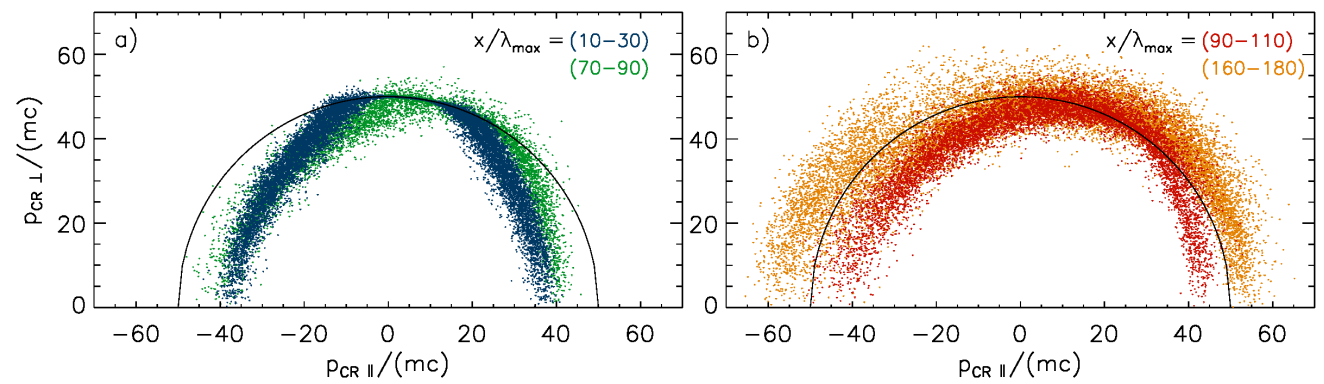

Figure 5: Phase-space distributions of CRs in the simulation frame and at $t \gamma_{\max }=26.9$. Distributions are shown for four color-marked regions defined in Figs. 2 and 4. Thin semicircles correspond to the initial monoenergetic isotropic distribution.

beam with negative momenta for $x / \lambda_{\max } \lesssim 90$. They cause substantial heating of the incoming plasma electrons and possibly also modify the magnetic-field structure through, e.g., ion-beam streaming instabilities.

\subsection{Cosmic ray scattering}

Figure 5 shows phase-space distributions of CRs in the four regions marked in Figs. 2 and 4. A degree of CR scattering and anisotropy introduced by the instability is evident. Most of the scattering is inelastic and occurs in the turbulent electric fields, thus modifying CR energy distribution.

Deeper insight into CR scattering is provided by Figure 6 that demonstrates the evolution of kinetic-energy and the $x$-component of sample CR trajectories. Scattering in energy commences at time $t \gamma_{\max } \approx 8$, when the amplitude of magnetic turbulence becomes comparable to the strength of the homogeneous field, $\delta B / B_{\| 0} \gtrsim 1$ (Fig. 3). The mean kinetic energy increases from the initial $\Gamma-1=49$ to $\sim 50$, because of the bulk energy transfer from the ambient plasma. One can note, that CR particles traveling in the positive $x$-direction are accelerated (particles No. 1 and 2), whereas CRs moving in the opposite direction are decelerated (particle No. 6). CRs that change their motion direction due to scattering correspondingly gain or lose energy (particles No. 3, 4, and 5). The scattering process requires a particle to reside in the region of strong turbulence, and hence energy fluctuations are seen at times $t \gamma_{\max } \gtrsim 10$ and locations $x \gtrsim 80 \lambda_{\max }$. These characteristics
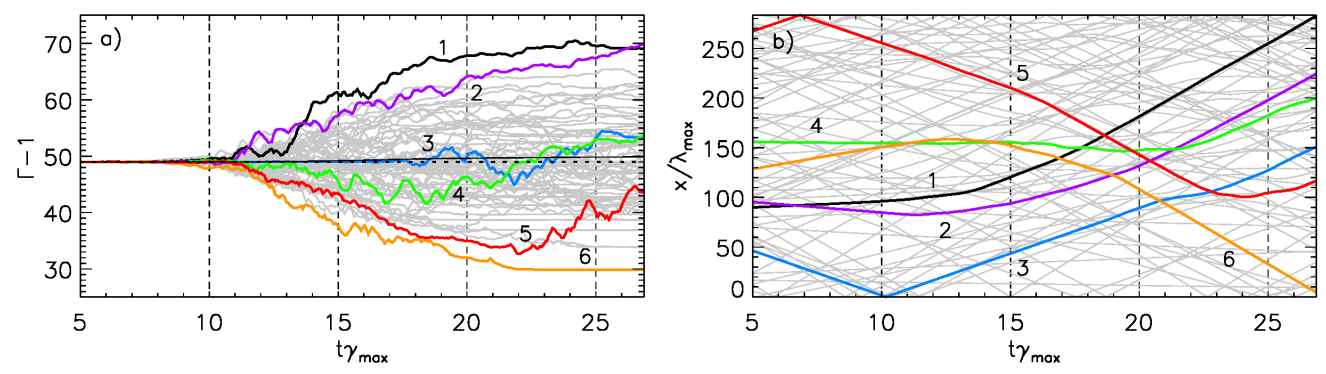

Figure 6: Time evolution of the kinetic energies (a) and $x$-coordinates (b) for typical CRs. Selected particles are numbered from 1 to 6 and marked with color. Gray background curves correspond to 200 randomly chosen CRs. The dotted horizontal line at $\Gamma-1=49$ marks the initial CR kinetic energy. The thin solid black line in the panel (a) shows the mean kinetic energy averaged for all CRs. 

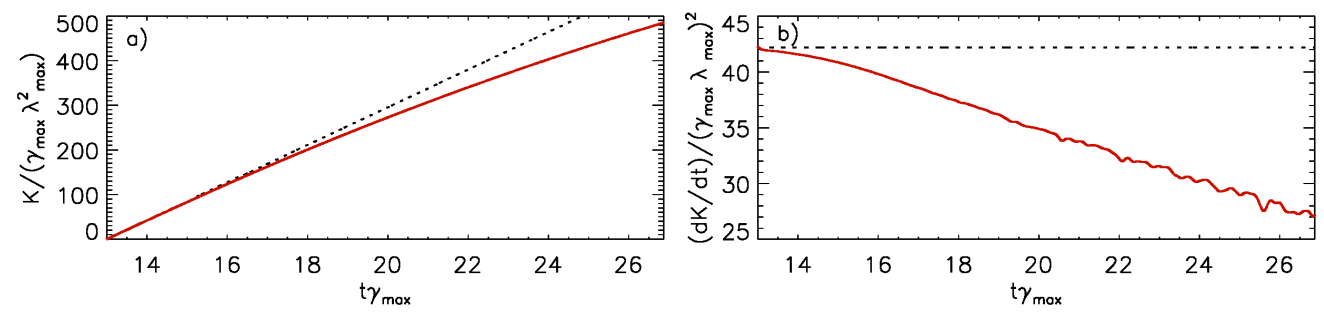

Figure 7: Running diffusion coefficient (a) of cosmic rays, $K(t)$, and its time derivative (b), $d K(t) / d t$, calculated for times $13 \leq t \gamma_{\max } \leq 26.9$. Dotted lines show the initial trends.

of CR scattering result from the structure of electric fields associated with the motion of turbulent magnetic field, in particular, the presence of a non-zero $E_{x}$ component in the turbulence region.

$\mathrm{CR}$ motion in magnetic turbulence resembles diffusion in space. We consider a running diffusion coefficient as $K(t)=\frac{1}{4 t}\left\langle\left(x-x_{0}\right)^{2}\right\rangle+\left\langle\left(y-y_{0}\right)^{2}\right\rangle$, where $x_{0}$ and $y_{0}$ are the initial particle locations. The coefficient $K(t)$ and its time derivative $d K(t) / d t$ are shown in Figures $7 \mathrm{a}$ and $7 \mathrm{~b}$, respectively. They are calculated from time $t \gamma_{\max }=13$, when turbulence just reached a saturation, till the end of simulation $t \gamma_{\max }=26.9$. One can see that CR motion is not truly diffusive as the coefficient does not reach constancy $K(t)=$ const. However, assuming the derivative in Fig. $7 \mathrm{~b}$ to be linear and extrapolating to $d K(t) / d t=0$, we can estimate the time, $t_{\text {diff }}$, needed for CRs to reach the diffusion regime, i.e., $K\left(t_{\text {diff }}\right)=$ const. This time is $t_{\text {diff }} \gamma_{\max } \approx 36.5 \pm 1$, and corresponds to the true diffusion coefficient $D=K\left(t_{\text {diff }}\right)=(770 \pm 20) \lambda_{\max }^{2} \gamma_{\max }$.

One can try to describe the diffusion of CRs in terms of the Bohm diffusion. The Bohm diffusion coefficient is $D_{\mathrm{B}}=r_{\mathrm{CRg}, \mathrm{f}}^{2} / \tau$, where $r_{\mathrm{CRg}, \mathrm{f}}$ is the $\mathrm{CR}$ gyro-radius in the amplified magnetic field and $\tau$ is the mean time between scatterings. Substituting the corresponding parameters into this formula, we obtain $D_{\mathrm{B}} \approx 740 \lambda_{\max }^{2} \gamma_{\max }$, that is close to value of $D$ estimated directly from the simulation results. Thus, the spatial CR scattering in the turbulent region is compatible with Bohm diffusion, when corrected for the coherence scale of magnetic turbulence.

\subsection{Plasma heating}
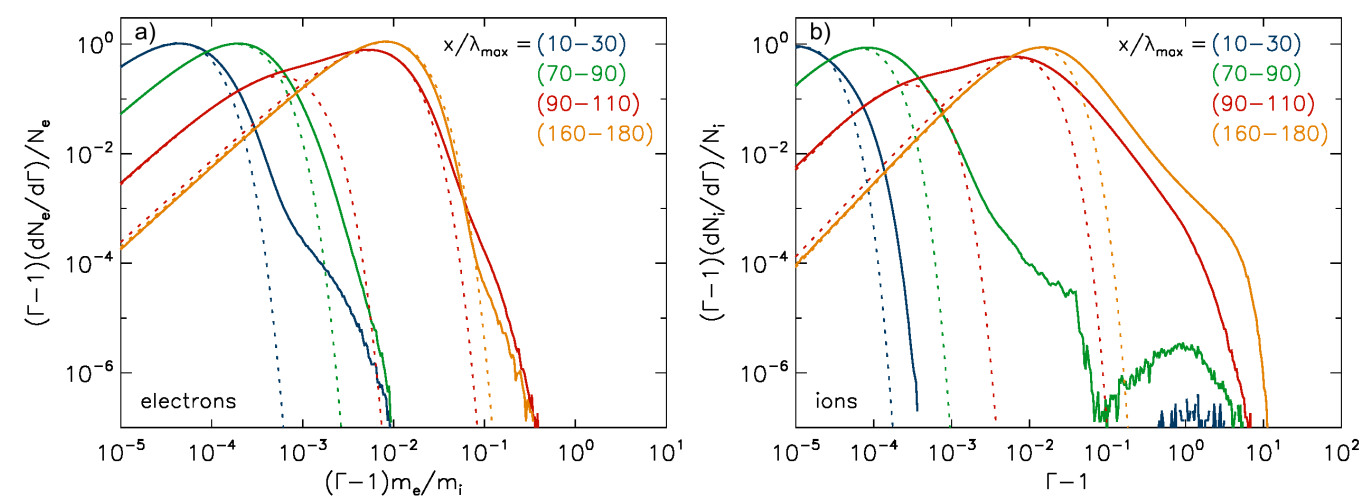

Figure 8: Kinetic-energy spectra of electrons (a) and ions (b) in the four regions marked in Figs. 2 and 4. The spectra are normalized and calculated at $t \gamma_{\max }=26.9$ in the local plasma frame. The electron energy is given in units of the ion rest-mass energy. Dotted lines show relativistic Maxwellian fits. 
As noted, nonlinear electromagnetic turbulence results in the significant heating of the background plasma. Ambient electron and ion spectra are shown in Figure 8. The particles are successively heated as they stream through the background of CRs, exciting the non-resonant instability. The ion spectra in the low-amplitude regions at $x / \lambda_{\max }=10-30$ (blue line) and $x / \lambda_{\max }=70-90$ (green line) in addition show components produced by ions reflected from the shock-like compression structure, as demonstrated above. These reflected ions also influence the electrons that formation tails in corresponding electron spectra. Effective heating takes place at $x / \lambda_{\max } \gtrsim 90$ (red and yellow lines). There is a bulk thermal equipartition between electrons and ions, but while electrons are largely relaxed to a Maxwellian distribution, the ions show prominent supra-thermal tails. The heating mechanism is rather similar for both particle species, but for ions another process enables their further energization beyond the thermal pool. This process is stochastic scattering in the turbulent convective electric fields.

Typical trajectory of a high-energy ion, accelerated to $\Gamma-1 \approx 8$, is marked on $2 \mathrm{D}$-maps presented in Figure 9. Detailed analysis of its energy evolution reveals an excellent correlation between gains and losses measured in the simulation frame and the work of the turbulent electric field $q(\mathbf{v} \cdot \mathbf{E})$. Although the work in the $E_{y}$ and $E_{z}$ fields averages to zero and results in pitch-angle scattering, the work in $E_{x}$ is on average positive and results in quasi-continuous energy gain. This behaviour resembles that described above for CR ions. Note, that scattering of supra-thermal elec-
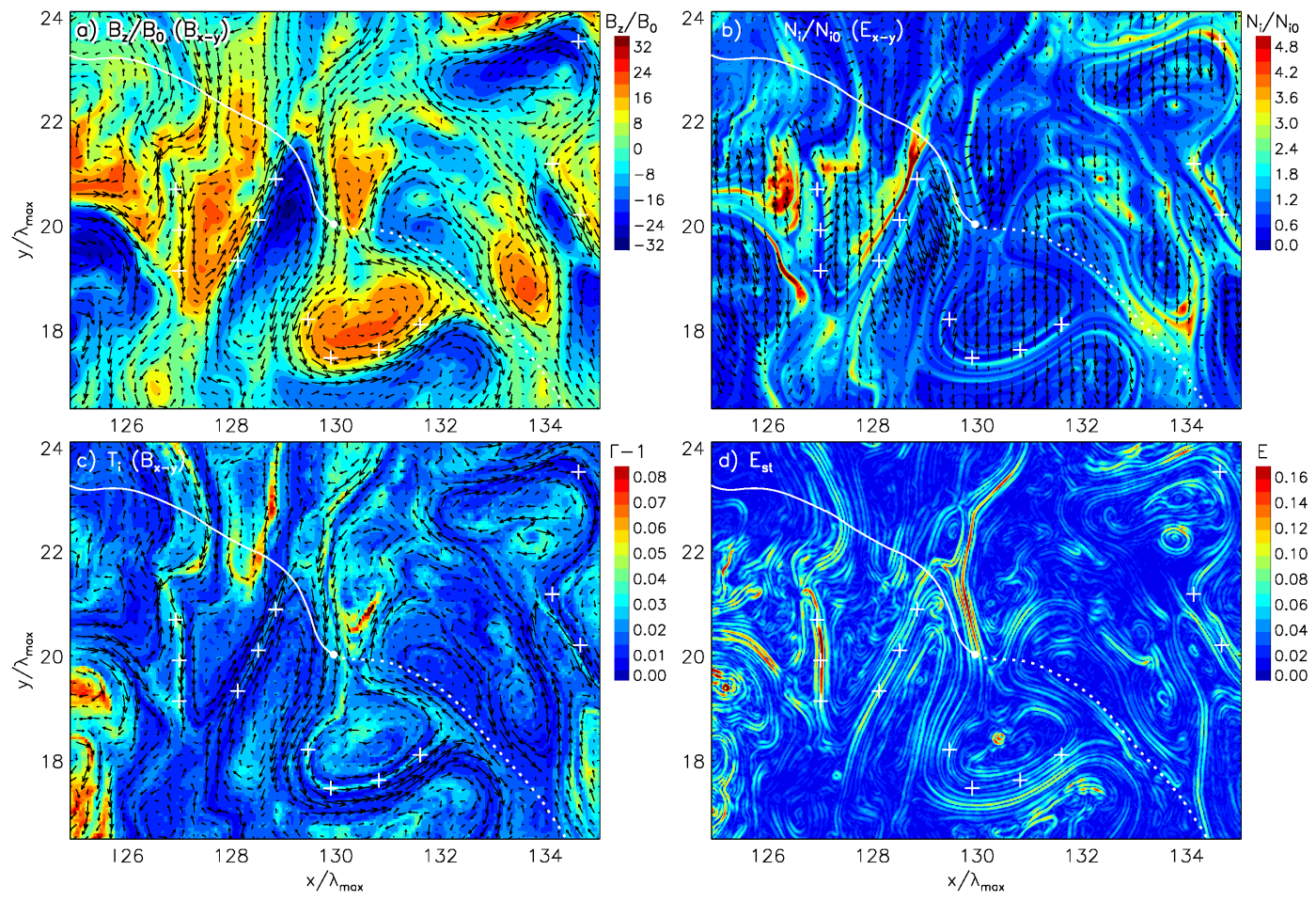

Figure 9: Distribution of the normalized magnetic-field amplitude $B_{z} / B_{0}$ (a), normalized ion number density $N_{i} / N_{i 0}$ (b), the mean kinetic energy (temperature) of ions (c), and charge-separation electrostatic field (d), at $t \gamma_{\max }=15$. Linear scales are used in all maps. In-plane magnetic-field lines (a, c) and in-plane electric-field lines (b) are overlaid. The white crosses are intended to facilitate cross-correlation. One example of an ion trajectory is shown as white solid line for times $t \gamma_{\max } \lesssim 15$ and as dotted line for later times. 
trons proceeds in a similar way, but due to small electron mass and high amplitude of turbulence all electric-field components can provide locally strong acceleration or deceleration of the electrons.

Detailed analysis of the microphysics of plasma heating can be performed by studying the turbulence structure in a small portion of the computational box (Fig. 9). Comparing ion distribution (Fig. 9b) with the temperature map (Fig. 9c), one can note that there is a correlation between the regions of high ion temperature and the regions of low plasma density. The electron temperature largely follows that of the ions. Such anticorrelation indicates that plasma heating does not result from adiabatic compression in turbulently moving plasma parcels. On the other hand, locations with high plasma temperature coincide with regions of large-amplitude electrostatic field (Fig. 9d). This shows that heating in the nonresonant large-amplitude turbulence is a non-adiabatic process.

\section{Conclusions}

Our results support the notion that the non-resonant Bell's instability can generate significant magnetic-field amplification in far upstream precursors of young SNR shocks. When combined with additional field production through instabilities operating on larger spatial scales, in close proximity to the shock front and/or at the shock itself, this process may be able to account for the level of magnetic-field amplification inferred from SNR observations. Similar conclusions may apply to shocks in relativistic jets and gamma-ray bursts. Electromagnetic turbulence thus produced can provide suitable conditions for particle acceleration to PeV energies at shocks in young SNRs.

\section{Acknowledgements}

This work has been supported by Narodowe Centrum Nauki through research projects DEC2011/01/B/ST9/03183 (J.N.) and DEC-2013/10/E/ST9/00662 (O.K., J.N. and A.B.). M.P. acknowledges support through grant PO 1508/1-2 of the Deutsche Forschungsgemeinschaft. The numerical experiment has been performed on the Prometheus system at ACC Cyfronet AGH. Part of the numerical work was conducted on resources provided by The North-German Supercomputing Alliance (HLRN) under project bbp00003 and also at the Pleiades facility at the NASA NAS.

\section{References}

[1] A. R. Bell, 2004, MNRAS, 353, 550

[2] V. N. Zirakashvili, V. S. Ptuskin, H. J. Voelk, 2008, ApJ, 678, 255

[3] K. M. Schure, A. R. Bell, 2013, MNRAS, 435, 1174

[4] A. Beresnyak, H. Li, 2014, ApJ, 788, 107

[5] S. G. Lucek, A. R. Bell, 2000, Ap\&SS, 272, 255

[6] L. Gargaté, R. A. Fonseca, J. Niemiec, et al., 2010, ApJ Lett., 711, L127

[7] J. Niemiec, M. Pohl, T. Stroman, K.-I. Nishikawa, 2008, ApJ, 684, 1174

[8] M. A. Riquelme, A. Spitkovsky, 2009, ApJ, 694, 626

[9] T. Stroman, M. Pohl, J. Niemiec, 2009, ApJ, 706, 38

[10] O. Kobzar, J. Niemiec, M. Pohl, A. Bohdan, 2017, MNRAS, 469, 4985 